\title{
Metasurfaces with Asymmetric Optical Transfer Functions for Optical Signal Processing
}

\author{
T. J. Davis and A. Roberts \\ School of Physics, University of Melbourne, Parkville Victoria 3010, Australia \\ D. E. Gómez \\ Centre of Excellence for Exciton Science, RMIT University, Melbourne Victoria 3000, Australia \\ F. Eftekhari \\ Melbourne Centre for Nanofabrication, Clayton Victoria 3168, Australia
}

\begin{abstract}
Metasurface thin-films created from arrays of structured optical elements have been shown to perform spatial filtering of optical signals. To extend their usefulness it is important that the symmetry of their response with changes to the in-plane wavevector $\mathbf{k}_{p} \rightarrow-\mathbf{k}_{p}$ can be tailored or even dynamically tuned. In this work we use a general theory of metasurfaces constructed from non-diffracting arrays of coupled metal particles to derive the optical transfer function and identify the physical properties essential for asymmetry. We validate our theory experimentally showing how the asymmetric response of a two-dimensional (planar) metasurface can be optically tuned. Our results set the direction for future developments of metasurfaces for optical signal processing.
\end{abstract}

PACS numbers: 78.67.Pt, 42.30.Lr, 42.30.Kq, 73.20.Mf

Metasurfaces are artificial thin-films created from subwavelength arrays of structured elements engineered to respond in ways not found in natural materials [1-3]. An optical metasurface with a transmission that depends on the wavevector $\mathbf{k}$ of the incident light field has potential as a highly compact spatial frequency filter [4-6]. A spatial filter performs optical signal processing by modifying the spatial Fourier harmonics of an image to highlight edges or remove specific features [7], a process akin to performing mathematical operations on the images. Likewise, difference operations or derivatives of the optical wavefront by metasurfaces enhance edges, make phase gradients visible $[8,9]$ or can be combined to form second derivatives and Laplacian operations. [10,11] Recently it was suggested that multilayers of metasurfaces could be used for forward and inverse optical Fourier transforms [12] enabling complex mathematical operations such as differentiation and integration $[13-15]$ to be performed in Fourier space [16] as well as solving differential equations. [17] Such multilayer metasurfaces can form asymmetries such that the transmittance depends on which face of the metasurface is illuminated. [18, 19]

The properties of an optical device can be described by the optical transfer function (OTF), which is the Fourier transform of the amplitude point-spread function of an optical system [20]. The OTF represents the response to plane-waves at different angles of incidence, providing a useful representation of the properties of optical metasurfaces [5]. When the response arises from a periodicity greater than the wavelength of light, the OTF becomes a nonlocal representation in $k$-space [6] and the device will exhibit diffraction. Metasurfaces that impose wavelength-scale periodic phase gradients across the transmitted beam [21-24] are equivalent to phase grat- ings [25] that preferentially diffract into a single order, giving the appearance of refraction that disobeys Snell's law. [26] Such diffractive phase surfaces, or holographic filters [7], are well-known and form the basis of computer generated holograms [27] with volume holograms demonstrating optical differentiation [28]. Spatial filtering exploiting the $k$-dependence of diffracting materials was used some decades ago to realise phase contrast imaging with hard x-rays $[29,30]$ with imaging properties also described using the OTF [31]. A synthesis between metasurfaces and diffration gratings was shown experimentally to redirect a light beam under all-optical control [32], giving the appearance of an optically-controlled blaze on the grating.

To be useful in optical signal processing, it is important that metasurfaces can replicate a large class of spatial filters. Filtering low spatial frequencies requires a null response at normal incidence, where $\mathbf{k} \cdot \mathbf{r}_{p}=0$ for vectors $\mathbf{r}_{p}$ in the plane, a property achievable using dark mode resonances $[4,5,33]$. More difficult is creating a metasurface with an asymmetric response that distinguishes between $-\mathbf{k} \cdot \mathbf{r}_{p}$ and $+\mathbf{k} \cdot \mathbf{r}_{p}$. Here we derive the physical conditions required for the asymmetric response of two dimensional non-diffracting metasurfaces, where the arrays of structured elements have subwavelength periodicity. We derive the OTF for an arbitrary arrangement of resonant nanostructures and classify terms based on their symmetry. This approach highlights the physical properties that are essential for asymmetry and lays the foundations for more complex metasurface designs. It was argued recently on the basis of reciprocity that twodimensional surfaces cannot exhibit such asymmetry because they are mirror-symmetric [6]. However the conditions we derive, necessary for asymmetry, include the 
(a)

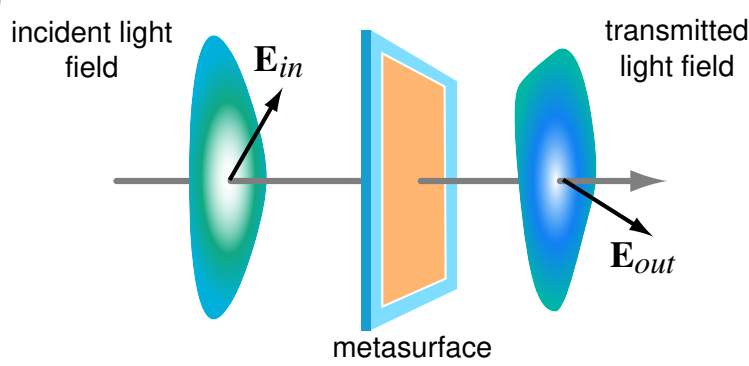

(b)

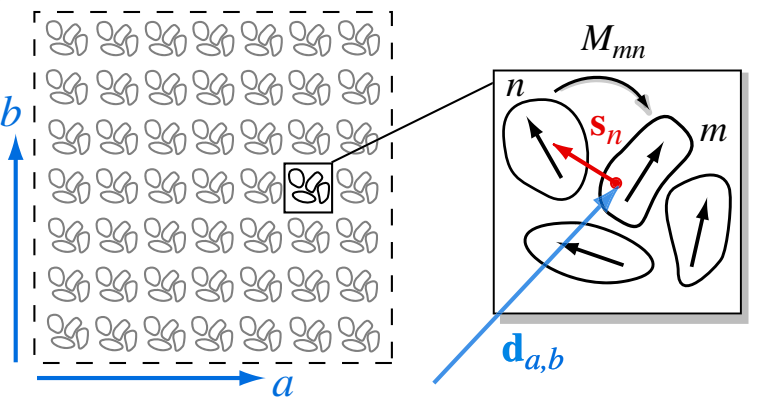

FIG. 1: The metasurface concept for spatial filtering. (a) The incident light field has a complicated $k$-space decomposition and polarization distribution $\mathbf{E}_{i n}$ that is transformed by the metasurface into another $k$-space and distribution $\mathbf{E}_{\text {out }}$; (b) The metasurface consists of an array of identical cells (indexed by integers $(a, b)$ ) composed of coupled resonant nanoparticles. The centre of the unit cell is located by the vector $\mathbf{d}_{a, b}$ and a particle in the cell by vector $\mathbf{s}_{n}$ relative to the cell centre. The arrows on each particle represent the dipole moments of the resonant modes.

effects of polarization and are precisely those that violate scalar reciprocity. We demonstrate asymmetric behavior experimentally with a non-diffracting metasurface and show how it can be selected optically.

The OTF $\overline{\mathcal{O}}\left(k_{x}, k_{y}\right)$ of a metasurface is the complex function that maps the incident electric field to the transmitted field (Fig. 1). It describes the optical response in the $x-y$ plane to the incident wavevector $\mathbf{k}_{I}=\left(k_{x}, k_{y}, k_{z}\right)$. The OTF can be written as a matrix to account for changes in the polarization of the transmitted light field [5]. We base our derivation of the OTF on the electrostatic eigenmode method for describing localised surface plasmon (LSP) resonances in subwavelength coupled systems. This method provides a simple algebra that encompasses most of the physics of nanoscale resonant systems $[35,36]$. The amplitude $a_{n}$ of the LSP excited by the electric field $\mathbf{E}_{0} e^{i \mathbf{k}_{I} \cdot \mathbf{r}_{n}}$ incident on a single plasmonic particle located at position $\mathbf{r}_{n}$ is $a_{n}=$ $f_{n}(\omega) \mathbf{p}_{n} \cdot \mathbf{E}_{0} e^{i \mathbf{k}_{I} \cdot \mathbf{r}_{n}}$ where $f_{n}(\omega) \approx-A_{n} /\left(\omega-\omega_{n}+i \Gamma_{n} / 2\right)$ describes the frequency dependence about the resonance at frequency $\omega_{n}$ with a full width at half maximum $\Gamma_{n}$. The vector $\mathbf{p}_{n}$ is proportional to the dipole moment of the resonance and $\mathbf{k}_{I}$ is the incident wavevector.

In the presence of $N-1$ other nanostructures within the cell or meta-atom of the metasurface, the electric fields of the LSPs couple, modifying the excitation amplitudes $a_{n}$. The new amplitudes $\tilde{a}_{m}$ can be written as linear combinations of the old ones, $\tilde{a}_{m}=\sum_{n}^{N} M_{m n} a_{n}$ where $M_{m n}$ is a matrix that mixes amplitudes together (Fig. 1(b)). The dipole moment of this excitation is $\tilde{a}_{m} \mathbf{p}_{m}$. The electric field scattered into the far field in direction $\hat{r}$ then takes the form [5]

$$
\mathbf{E}_{m}=\sum_{n}^{N} \overline{\mathbf{e}} \cdot \mathbf{p}_{m}\left(\mathbf{p}_{n} \cdot \mathbf{E}_{0}\right) C_{m n} e^{i \mathbf{k}_{I} \cdot \mathbf{r}_{n}-i \mathbf{k}_{s} \cdot \mathbf{r}_{m}},
$$

where we have set $\overline{\mathbf{e}}=k^{2} e^{i k r}(1-\hat{r} \hat{r}) / 4 \pi \epsilon_{0} r$. The coupling term $C_{m n}=M_{m n} f_{n}(\omega)=\left[\delta_{m n}-f_{m}(\omega) G_{m n}\right]^{-1} f_{n}(\omega)$, which depends on the matrix inverse $[\ldots]^{-1}$, is symmetric on interchange of the indices, since the dipole interaction $G_{m n}$ of the evanescent fields between particles that we consider here is symmetric $G_{m n}=G_{n m}$ [36].

We now consider the metasurface created from a periodic array of meta-atoms and show that metasurfaces with subwavelength periodicities behave like uniform thin films. We assume identical unit cells of $N$ coupled metal particles in the $x-y$ plane. If the array period is $\mathbf{d}_{a, b}=a d_{x} \hat{x}+b d_{y} \hat{y}$ with $a$ and $b$ integers, $d_{x}$ the array period in the $\hat{x}$ direction and $d_{y}$ the period for $\hat{y}$, then we can write $\mathbf{r}_{m}=\mathbf{d}_{a, b}+\mathbf{s}_{m}$ where $\mathbf{s}_{m}$ is a small vector from the centre of the unit cell to the $m$-th nanostructure and the sum over $m$ is only over the structures within the unit cell (Fig. 1 (b)). Then we sum Eq. (1) to yield the total far-field electric field

$$
\mathbf{E}=\sum_{a, b} \sum_{m, n} \overline{\mathbf{e}} \cdot \mathbf{p}_{m}\left(\mathbf{p}_{n} \cdot \mathbf{E}_{0}\right) C_{m n} e^{i \mathbf{k}_{I} \cdot \mathbf{s}_{n}-i \mathbf{k}_{s} \cdot \mathbf{s}_{m}-i \mathbf{q}_{s} \cdot \mathbf{d}_{a, b}},
$$

where $\mathbf{q}_{s}=\mathbf{k}_{s}-\mathbf{k}_{I}$ is the scattering vector. Since all unit cells in the periodic array are identical the sum over the array separates into two factors that are generally very small, except when $\mathbf{q}_{s} \cdot \hat{x} d_{x}=2 n \pi$ or $\mathbf{q}_{s} \cdot \hat{y} d_{y}=2 m \pi$ where $n$ and $m$ are integers [5]. These two expressions are equivalent to the diffraction equation for two dimensions. For example, writing $\mathbf{k}_{I}=k\left(\hat{x} \sin \theta_{I}-\hat{z} \cos \theta_{I}\right)$, and likewise for $\mathbf{k}_{s}$, then $\mathbf{q}_{s} \cdot \hat{x} d_{x}=k d_{x}\left(\sin \theta_{s}-\sin \theta_{I}\right)=2 n \pi$ or $\sin \theta_{s}=\sin \theta_{I}+n \lambda / d_{x}$, which is the diffraction equation with $k=2 \pi / \lambda$. This equation has been described erroneously as a generalization of Snell's Law. [21]

For periodicities much smaller than the wavelength of light, the dominant scattering occurs only for $n=m=0$ for which $\mathbf{q}_{s} \cdot \hat{d}=0$ where $\hat{d}$ is a vector in the $x-y$ plane. This condition implies that $\mathbf{k}_{s}=\mathbf{k}_{I}$ so that the incident beam is merely transmitted through the periodic array (the zero diffraction order). Alternatively we can have specular reflection since this also satisfies $\left(\mathbf{k}_{s}-\mathbf{k}_{I}\right) \cdot \hat{d}=0$.

The total scattered electric field is then given by

$$
\begin{aligned}
\mathbf{E} & =N_{a} N_{b} \sum_{m, n} \overline{\mathbf{e}} \cdot \mathbf{p}_{m}\left(\mathbf{p}_{n} \cdot \mathbf{E}_{0}\right) C_{m n} e^{i \mathbf{k}_{I} \cdot\left(\mathbf{s}_{n}-\mathbf{s}_{m}\right)} \\
& =N_{a} N_{b} \overline{\mathbf{e}} \cdot \overline{\mathcal{O}}\left(k_{x}, k_{y}\right) \cdot \mathbf{E}_{0},
\end{aligned}
$$


for $N_{a} N_{b}$ unit cells in the array. The optical transfer function $\overline{\mathcal{O}}\left(k_{x}, k_{y}\right)$ is

$$
\overline{\mathcal{O}}\left(k_{x}, k_{y}\right)=\sum_{m, n} \mathbf{p}_{m} \mathbf{p}_{n} C_{m n} e^{i \mathbf{k}_{I} \cdot\left(\mathbf{s}_{n}-\mathbf{s}_{m}\right)}
$$

that involves the outer product of the two vectors $\mathbf{p}_{m} \mathbf{p}_{n}$ - in effect, $\mathbf{p}_{m}$ is operated on from the left and $\mathbf{p}_{n}$ is operated on from the right. For simplicity we have omitted $\overline{\mathbf{e}}$ from the definition of the optical transfer function, although it can be easily included as required. The outer product of two vectors such as $\mathbf{p}_{m} \mathbf{p}_{n}$ is known as a dyadic. In the context of the OTF the dyadic represents a change in the direction of polarization of the incident beam and the factor $\mathbf{p}_{m} \mathbf{p}_{n}$ is equivalent to a component of the Jones matrix $\mathbb{M}$ in optics [37]. For example, an incident beam with amplitude $\mathbf{E}_{I}$ is scattered with polarization related to $\mathbf{p}_{m}\left(\mathbf{p}_{n} \cdot \mathbf{E}_{I}\right)$.

To make asymmetric dependences on $\mathbf{k}_{I}$ explicit, we note that the coupling is symmetric $C_{m n}=C_{n m}$ and rewrite the double sum in Eq. (4) as

$$
\begin{aligned}
\overline{\mathcal{O}}\left(k_{x}, k_{y}\right) & =\sum_{m} p_{m}^{2} \hat{p}_{m} \hat{p}_{m} C_{m m}+\sum_{m, n>m} C_{m n} p_{m} p_{n} \\
& \times\left[\left(\hat{p}_{m} \hat{p}_{n}+\hat{p}_{n} \hat{p}_{m}\right) \cos \mathbf{k}_{I} \cdot\left(\mathbf{s}_{n}-\mathbf{s}_{m}\right)\right. \\
& \left.+i\left(\hat{p}_{m} \hat{p}_{n}-\hat{p}_{n} \hat{p}_{m}\right) \sin \mathbf{k}_{I} \cdot\left(\mathbf{s}_{n}-\mathbf{s}_{m}\right)\right],
\end{aligned}
$$

where $\mathbf{p}_{n}=p_{n} \hat{p}_{n}$ separates out the magnitude $p_{n}$ of the eigenmode dipole moment from its orientation $\hat{p}_{n}$. The only $k$-dependence of the OTF is through the phase factors in Eq. (5), with $\cos \mathbf{k}_{I} \cdot\left(\mathbf{s}_{n}-\mathbf{s}_{m}\right)$ being symmetric and $\sin \mathbf{k}_{I} \cdot\left(\mathbf{s}_{n}-\mathbf{s}_{m}\right)$ asymmetric under interchange $\mathbf{k}_{I} \rightarrow-\mathbf{k}_{I}$. In particular, an asymmetric response requires the coefficient in front of the sine term to satisfy $\hat{p}_{m} \hat{p}_{n} \neq \hat{p}_{n} \hat{p}_{m}$.

Thus we conclude that a metasurface consisting of an array of identical cells of subwavelength periodicity may demonstrate an asymmetric response only if the constituents in the cell have dipole resonances that are misaligned. This is the key result of our paper. This result contradicts the analysis of Kwon et al [6] who used a scalar form of reciprocity to argue that transmission asymmetry with the in-plane $k$-vector could be obtained only by breaking both horizontal and vertical mirror symmetry. However, the most general form of the reciprocity relation [38], attributed to de Hoop [34], requires the Jones matrix $\mathbb{M}_{f}$ of the forward propagation to equal the transpose of the matrix for reverse propagation $\mathbb{M}_{r}^{T}=\mathbb{M}_{f}$. The assumption in Ref. [6] is that the forward and reverse propagation are the same, implying that $\mathbb{M}_{f}^{T}=\mathbb{M}_{f}$. This condition is violated in Eq. (5) precisely when the Jones matrix components $\hat{p}_{m} \hat{p}_{n} \neq \hat{p}_{n} \hat{p}_{m}$ are unequal, which breaks the symmetry and destroys scalar reciprocity in our two-dimensional system. For example consider pairs of LSP modes with dipole moments misaligned and the incident light polarized. The

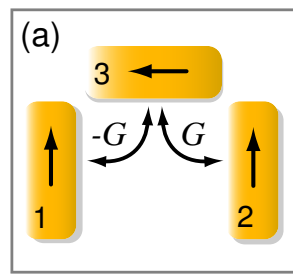

(c)
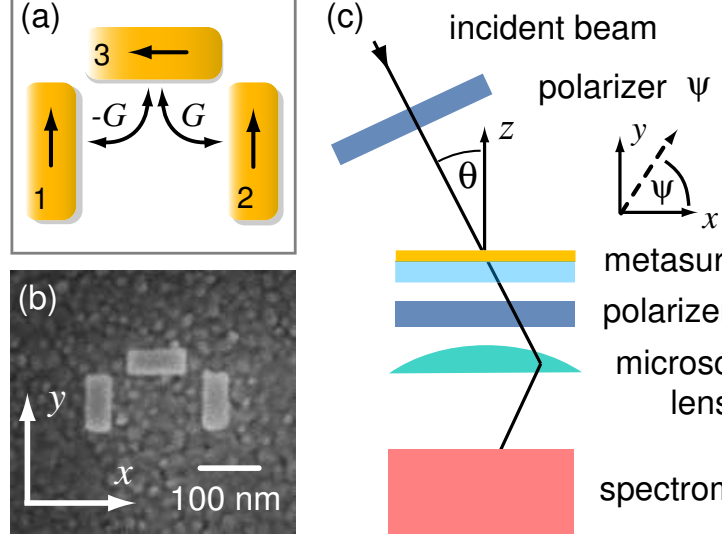

metasurface

polarizer $\hat{y}$

microscope

lens

spectrometer
FIG. 2: Experiment to demonstrate asymmetric optical transfer functions. (a) The plasmon "Wheatstone bridge" of three identical gold nanorods showing the predominant coupling. The arrows represent the induced dipole moments for the polarizer angle $90^{\circ}<\psi \leq 180^{\circ}$; (b) SEM image of an example circuit prior to etching away the underlying film; (c) the experiment configuration. The metasurface is a subwavelength periodic array of the structures in (b). The light beam is incident on the metasurface at angle $\theta$.

transfer function is $\hat{P}_{o} \cdot \overline{\mathcal{O}}\left(k_{x}, k_{y}\right) \cdot \hat{P}_{i}$ where an input linear polarizer $\hat{P}_{i}$ sets the direction of the incident field and an output linear polarizer $\hat{P}_{o}$ controls the measured field. If $\psi_{n}$ and $\phi_{n}$ are the angles between $\mathbf{p}_{n}$ and the incident and the exit polarizers respectively, then the term in brackets of Eq. (5) is

$$
2 f_{n}\left[a \cos \mathbf{k}_{I} \cdot\left(\mathbf{s}_{n}-\mathbf{s}_{m}\right)+i b \sin \mathbf{k}_{I} \cdot\left(\mathbf{s}_{n}-\mathbf{s}_{m}\right)\right],
$$

where $a=\left(\cos \phi_{m} \cos \psi_{n}+\cos \phi_{n} \cos \psi_{m}\right) / 2$ and $b=$ $\left(\cos \phi_{m} \cos \psi_{n}-\cos \phi_{n} \cos \psi_{m}\right) / 2$. This expression shows an asymmetry, provided $b \neq 0$.

To demonstrate that such asymmetric behaviour is physically possible we perform an experiment on a metasurface created from a subwavelength array of three identical nanorods supporting LSP resonances (Fig. 2(a)). This configuration mimics the Wheatstone Bridge (WB) circuit in electronics [39] that performs first-order difference operations enabling phase sensing [8]. Although such a design may not be optimum for optical signal processing, we use it here to demonstrate both symmetric and asymmetric response in the transmitted intensity controlled by the orientation of a polarizer. Such effects have not previously been reported.

The WB circuit consists of three gold nanorods, each $40 \mathrm{~nm}$ wide, $30 \mathrm{~nm}$ thick and $100 \mathrm{~nm}$ long (Fig. 2(b)) on a borosilicate glass substrate. Two parallel rods (labelled 1 and 2) are oriented in the $\hat{y}$ direction with a centreto-centre distance $d=200 \mathrm{~nm}$ and the third "bridge" nanorod is oriented in $\hat{x}$ with its bottom edge aligned with the tops of the two parallel rods. This placement controls the strength $G$ of the coupling between the rods that affects the degree of asymmetry in the response. 
The metasurface consists of arrays of WB circuits with a period of $400 \mathrm{~nm}$ in both directions. The experiment is performed using the configuration in Fig. 2(c). Collimated white light passed though a polarizer, aligned at angle $\psi$ to nanorod 3 , is incident on the metasurface at an angle $\theta$ to the surface normal, giving an in-plane wave vector $k_{x}=k \sin \theta$. The transmitted light is filtered by a second polarizer, aligned with the parallel rods, and then measured by a spectrometer.

An example transmission spectrum is shown in Fig. $3(\mathrm{a})$, measured at three incidence angles $\theta$, expressed in terms of the in-plane wave vector $k_{x}$. The first polarizer was set to angle $\psi=45^{\circ}$. The spectra near the resonance at $\lambda=686 \mathrm{~nm}$ show increasing transmission as $k_{x}$ changes sign from negative to positive, providing a clear demonstration of an asymmetry in the metasurface response. The normalised transmission at the resonance as a function of $k_{x}$ for four different polarizer angles is shown by experimental data points in Fig. 3(b). The direction of the asymmetry is controlled by the polarizer and changes sign when $\psi=45^{\circ} \rightarrow 135^{\circ}$.

The asymmetry can be modelled using the OTF of the metasurface. With $p=|\mathbf{p}|$ being the magnitude of the LSP eigenvector dipole moment and the resonance factor $f\left(\omega=\omega_{R}\right)=i 2 A / \Gamma \equiv i f_{R}$ being purely imaginary at resonance, the OTF from Eq. (4) (see the Supplement for a derivation and numerical simulations) is

$$
\begin{aligned}
\hat{P}_{o} \cdot \overline{\mathcal{O}}\left(k_{x}\right) \cdot \hat{P}_{i} & =\frac{i 2 p^{2} f_{R}}{1+2 f_{R}^{2} G^{2}}\left[\cos \psi \cos \theta f_{R} G \sin \left(k_{x} d / 2\right)\right. \\
& \left.+\sin \psi\left(1+2 f_{R}^{2} G^{2} \cos ^{2}\left(k_{x} d / 2\right)\right)\right],
\end{aligned}
$$

where the orientation of the input polarizer is $\hat{P}_{i}=$ $\cos \psi \cos \theta \hat{x}+\sin \psi \hat{y}$ and the output polarizer is $\hat{P}_{o}=\hat{y}$. The $\cos \theta$ term in $\hat{P}_{i}$ arises from the polarizer being attached to the tilt arm in the experiment, as shown in Fig. 2(c). The OTF contains a term antisymmetric in $k_{x}$, which is selected by $\psi=0$ and a term symmetric in $k_{x}$ obtained with $\psi=\pi / 2$. Since most experiments measure intensities proportional to $|\mathrm{OTF}|^{2}$, all negative quantities become positive and the asymmetry is not usually observed directly, as in the case of the WB experiments of Eftekhari et al [8] and described by Roberts et al [5]. However, the cross-term in the intensity depends on $2 \cos \psi \sin \psi=\sin 2 \psi$ that remains predominantly antisymmetric in $k_{x}$, selected with the polarizer at angles $\psi= \pm \pi / 4$. Indeed, the polarizer also determines the direction of the asymmetry with $k_{x}$ with a magnitude that depends on $f_{R} G$. The theoretical result as the square of Eq. (7) is included with the experimental results in Fig. 3(b). The theoretical curves are matched to the data by adjusting two parameters only, such that $f_{R} G=0.24$ and $2 p^{2} f_{R}=1.00$. For $\psi=45^{\circ}$ and $135^{\circ}$ the data show the expected asymmetry with $k_{x}$ and excellent agreement with theory. As discussed in the Supplement, we would (a)

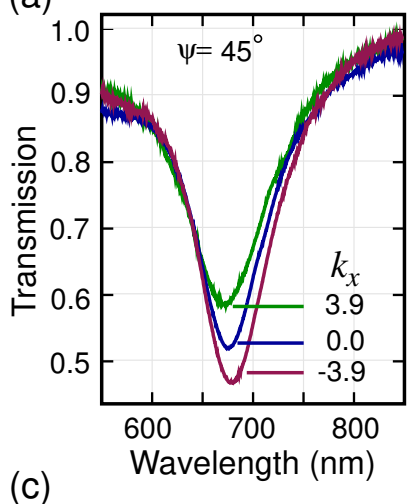

(c)
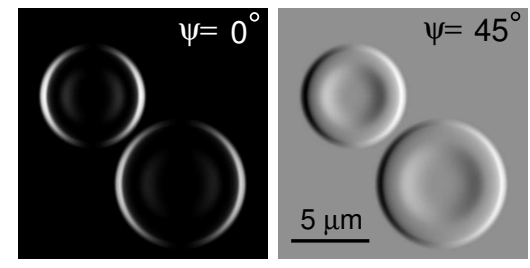

(b)

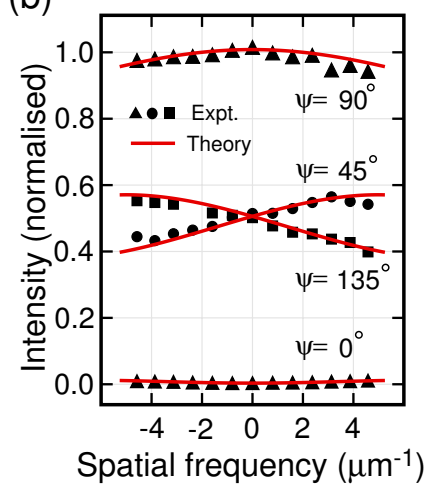

FIG. 3: (a) Experimentally measured spectra of light transmitted through the metasurface for three incidence angles, expressed as in-plane wave numbers $k_{x}=k \sin \theta$. The incident polarizer orientation was $\psi=45^{\circ}$; (b) The response of the metasurface to different spatial frequencies $k_{x}$ for four settings $\psi$ of the incident polarizer. The polarizer controls the symmetry of the response and the sign of the asymmetry for $\psi=45^{\circ}, 135^{\circ}$. The points are from experiment and solid lines are $\left|\hat{P}_{o} \cdot \overline{\mathcal{O}}\left(k_{x}\right) \cdot \hat{P}_{i}\right|^{2}$ derived from Eq. (7). (c) Simulations using Eq. (7) of the metasurface response to light from pure phase objects representing biological cells with optical phase excursions of $120^{\circ}$.

expect similar asymmetry with no output polarizer.

The asymmetry has its origin in the direction of the incident electric field on nanorod 3. Referring to Fig. 2 (a), for the polarizer set in the range $90^{\circ}<\psi \leq 180^{\circ}$, the induced dipole moment in nanorod 3 leads to positive coupling $G$ to nanorod 2 (dipoles adding) and negative coupling to nanorod 1 (dipoles opposing), that acts to drive the nanorods out of phase. This phase shift results in partial cancellation of the transmitted light at normal incidence $k_{x}=0$. An additional phase shift is applied to these nanorods by changing the angle of incidence (changing $k_{x}$ ) that either reduces or enhances the interference according to the sign of $k_{x}$. The situation is reversed when $0^{\circ} \leq \psi<90^{\circ}$ because the induced dipole moment on nanorod 3 is reversed which changes the direction of asymmetry with $k_{x}$. This behavior is consistent with numerical simulations shown in the Supplement.

With the OTF we can simulate the response of the metasurface to light from a pure phase object. Fig. 3(c) shows the simulated transmission images for light passing through a biological-like sample. The optical parameters for the sample are those from red blood cells in water [40]. Each image represents a field of view of $16 \mu \mathrm{m}$ and 
we assume the "optical system" has a spatial resolution of $400 \mathrm{~nm}$. It is apparent that the contrast changes with the orientation of the polarizer. The metasurface enables phase imaging with an asymmetric contrast similar to differential contrast imaging in more complex optical systems.

In this work we have demonstrate both symmetric and asymmetric behaviour of a plasmonic metasurface and provided physical criteria for creating more complex metasurface spatial frequency filters. When such subwavelength structures are combined with complex holographic-type diffraction patterns we expect to create highly versatile and compact devices for manipulating the information content in images.

This work was performed in part at the Melbourne Centre for Nanofabrication (MCN) in the Victorian Node of the Australian National Fabrication Facility (ANFF). The authors acknowledge funding through the Australian Research Council Discovery Projects Scheme (DP160100983) and Future Fellowship Scheme (FT140100514).

[1] A. Kildishev, A. Boltasseva, and V. Shalaev, Science 339, 1232009 (2013).

[2] Y. Zhao, X. Liu, and A. Al, Journal Of Optics 16, 123001 (2014).

[3] L. Zhang, S. Mei, K. Huang, and C. Qiu, Adv. Opt. Mater. 4, 818 (2016).

[4] A. Roberts, T. J. Davis, and D. E. Gómez, J. Opt. Soc. Am. B 34, D95 (2017).

[5] A. Roberts, D. E. Gómez, and T. J. Davis, J. Opt. Soc. Am. A 35, 1575 (2018).

[6] H. Kwon, D. Sounas, A. Cordaro, A. Polman, and A. Alù, Phys. Rev. Lett. 121, 173004 (2018).

[7] K. G. Birch, Rep. Prog. Phys. 35, 1265 (1972).

[8] F. Eftekhari, D. Gómez, and T. Davis, Opt. Lett. 39, 2994 (2014).

[9] Y. Hwang and T. Davis, App. Phys. Lett. 109, 10.1063 (2016).

[10] D. A. Bykov, L. L. Doskolovich, E. A. Bezus, and V. A. Soifer, Opt. Express 22, 25084 (2014).

[11] Y. Hwang, T. J. Davis, J. Lin, and X. Yuan, Opt. Express 26, 7368 (2018).

[12] A. Silva, F. Monticone, G. Castaldi, V. Galdi, A. Alù, and N. Engheta, Science 343, 160 (2014).

[13] A. Chizari, S. Abdollahramezani, M. V. Jamali, and J. A.
Salehi, Opt. Lett. 41, 3451 (2016).

[14] C. Dai, Z. Zhao, X. Li, and H. Yang, Phys. Lett. A 380, 3942 (2016).

[15] A. Youssefi, F. Zangeneh-Nejad, S. Abdollahramezani, and A. Khavasi, Opt. Lett. 41, 3467 (2016).

[16] A. Pors, M. Nielsen, and S. Bozhevolnyi, Nano Lett. 15, 791 (2015).

[17] W. Zhang, C. Qu, and X. Zhang, Journal Of Optics 18, 075102 (2016).

[18] A. Shevchenko, V. Kivijärvi, P. Grahn, M. Kaivola, and K. Lindfors, Phys. Rev. Applied 4, 024019 (2015).

[19] C. Pfeiffer and A. Grbic, Phys. Rev. Lett. 117, 077401 (2016).

[20] A. K. Ghatak and K. Thyagarajan, Fourier Optics II. Optical Transfer Functions (Plenum Press, New York, 1978), ISBN 978-1-4684-2360-0.

[21] N. Yu, P. Genevet, M. Kats, F. Aieta, J. Tetienne, F. Capasso, and Z. Gaburro, Science 334, 333 (2011).

[22] N. M. Estakhri and A. AlÙ, Phys. Rev. B 89, 235419 (2014).

[23] L. Huang, X. Chen, H. Mhlenbernd, G. Li, B. Bai, Q. Tan, G. Jin, T. Zentgraf, and S. Zhang, Nano Lett. 12, 5750 (2012).

[24] J. Li, S. Chen, H. Yang, J. Li, P. Yu, H. Cheng, C. Gu, H. Chen, and J. Tian, Adv. Func. Mater. 25, 704 (2015).

[25] R. Magnusson and T. K. Gaylord, J. Opt. Soc. Am. 68, 806 (1978).

[26] S. Larouche and D. R. Smith, Opt. Lett. 37, 2391 (2012).

[27] F. Wyrowski, J. Opt. Soc. Am. A 7, 961 (1990).

[28] S. K. Case, Opt. Lett. 4, 286 (1979).

[29] T. J. Davis, D. Gao, T. E. Gureyev, A. W. Stevenson, and S. W. Wilkins, Nature 373, 595 (1995).

[30] T. J. Davis, T. E. Gureyev, D. Gao, A. W. Stevenson, and S. W. Wilkins, Phys. Rev. Lett. 74, 3173 (1995).

[31] T. J. Davis, J. X-ray Sci. Technol. 6, 317 (1996).

[32] T. J. Davis, D. E. Gómez, and F. Eftekhari, Opt. Lett. 39, 4938 (2014).

[33] L. Wesemann, P. Achmari, K. Singh, E. Panchenko, T. D. James, D. E. Gómez, T. J. Davis, and A. Roberts, OSA Continuum 1, 727 (2018).

[34] A. T. de Hoop, Appl. Sci. Res. B 8, 135 (1959).

[35] T. J. Davis, K. C. Vernon, and D. E. Gómez, Phys. Rev. B 79, 155423 (2009).

[36] T. Davis and D. E. Gómez, Rev. Mod. Phys. 89, 011003 (2017).

[37] R. C. Jones, J. Opt. Soc. Am. 31, 488 (1941).

[38] R. J. Potton, Rep. Prog. Phys. 67, 717 (2004).

[39] T. Davis, K. Vernon, and D. Gómez, J. Appl. Phys. 106, 043502 (2009).

[40] M. Kinnunen, A. Kauppila, A. Karmenyan, and R. Myllylä, Biomed. Opt. Express 2, 1803 (2011). 


\section{University Library}

\section{- M M N E R VA A gateway to Melbourne's research publications}

Minerva Access is the Institutional Repository of The University of Melbourne

Author/s:

Davis, TJ;Eftekhari, F;Gomez, DE;Roberts, A

Title:

Metasurfaces with Asymmetric Optical Transfer Functions for Optical Signal Processing

Date:

2019-07-01

Citation:

Davis, T. J., Eftekhari, F., Gomez, D. E. \& Roberts, A. (2019). Metasurfaces with Asymmetric Optical Transfer Functions for Optical Signal Processing. PHYSICAL REVIEW LETTERS, 123 (1), https://doi.org/10.1103/PhysRevLett.123.013901.

Persistent Link:

http://hdl.handle.net/11343/243784 